\title{
Continuous measures of driving performance on an advanced office-based driving simulator can be used to predict simulator task failure in patients with obstructive sleep apnoea syndrome
}

\author{
Dipansu Ghosh, ${ }^{1}$ Samantha L Jamson, ${ }^{2}$ Paul D Baxter, ${ }^{3}$ Mark W Elliott ${ }^{1}$
}

\begin{abstract}
- Additional materials are published online only. To view these files please visit the journal online (http://dx.doi.org/ 10.1136/thoraxjnl-2011200699).

'Department of Respiratory Medicine, St James' University Hospital, Leeds, UK ${ }^{2}$ Safety and Technology Group, Institute for Transport Studies, University of Leeds, Leeds, UK ${ }^{3}$ Division of Biostatistics, LIGHT, Centre for Epidemiology and Biostatistics, University of Leeds, Leeds, UK
\end{abstract}

Correspondence to Dr Mark W Elliott, Consultant Respiratory Physician Department of Respiratory Medicine, Sleep and Non-invasive Ventilation Services, St James' University Hospital, Beckett Street, Leeds LS9 7TF, UK:

mark.elliott@leedsth.nhs.uk

Received 28 June 2011 Accepted 4 April 2012

Published Online First

5 May 2012

\section{ABSTRACT \\ Introduction Some patients with obstructive sleep} apnoea syndrome are at higher risk of being involved in road traffic accidents. It has not been possible to identify this group from clinical and polysomnographic information or using simple simulators. We explore the possibility of identifying this group from variables generated in an advanced PC-based driving simulator. Methods All patients performed a $90 \mathrm{~km}$ motorway driving simulation. Two events were programmed to trigger evasive actions, one subtle and an alert driver should not crash, while for the other, even a fully alert driver might crash. Simulator parameters including standard deviation of lane position (SDLP) and reaction times at the veer event (VeerRT) were recorded. There were three possible outcomes: 'fail', 'indeterminate' and 'pass'. An exploratory study identified the simulator parameters predicting a 'fail' by regression analysis and this was then validated prospectively.

Results 72 patients were included in the exploratory phase and 133 patients in the validation phase. 65 (32\%) patients completed the run without any incidents, 45 $(22 \%)$ failed, 95 (46\%) were indeterminate. Prediction models using SDLP and VeerRT could predict 'fails' with a sensitivity of $82 \%$ and specificity of $96 \%$. The models were subsequently confirmed in the validation phase. Conclusions Using continuously measured variables it has been possible to identify, with a high degree of accuracy, a subset of patients with obstructive sleep apnoea syndrome who fail a simulated driving test. This has the potential to identify at-risk drivers and improve the reliability of a clinician's decision-making.

\section{INTRODUCTION}

On average, patients with obstructive sleep apnoea syndrome (OSAS) are at increased risk of being involved in a road traffic accident, but not all patients with OSAS are unsafe drivers. Currently advice about an individual's fitness to drive is based on the severity of the sleep-disordered breathing and daytime sleepiness, and their account of their driving. ${ }^{1-4}$ Although there is a trend towards increased likelihood of accidents with more severe sleep-disordered breathing, these are not sufficiently robust data on which to base decisions for an individual. ${ }^{5}$ There are conflicting data about the relationship between perceived

\section{Key messages}

What is the key question?

- Is it possible to identify a group of patients with obstructive sleep apnoea syndrome (OSAS) who are potentially at risk of being involved in road traffic accidents using an advanced office-based driving simulator?

What is the bottom line?

- It is possible to identify with a high degree of accuracy a subset of patients with OSAS who are likely to fail a simulated driving scenario, in a way that has 'street credibility' using continuously recorded data from the simulator.

Why read on?

- Using subtle parameters of which the subjects remain unaware, this simulator provides hope for a much needed objective test to help clinicians advise patients with OSAS about driving.

sleepiness and the likelihood of being involved in an accident ${ }^{67}$ and between subjective and objective tests for increased daytime sleepiness. ${ }^{8-10}$ Driving requires alertness and also complex integrated higher cortical function; patients with OSAS may have neurological damage, which may impact on driving. ${ }^{11}$ Driving may therefore be impaired for reasons other than those just related to maintenance of alertness. The advice that a patient will receive about driving will also depend on their doctor's attitude to risk and this is likely to be inconsistent in the absence of robust objective criteria. There is therefore a need for an objective test, which can help to inform the advice that clinicians give to patients with OSAS.

Any such test should evaluate as many aspects as possible of all the functions needed for safe driving, and not just alertness. Performing studies during real driving is not feasible. PC-based driving simulators provide objective data and previous studies have shown that patients with OSAS tend to perform worse than normal subjects on driving simulators, but there is considerable overlap. ${ }^{12-14}$ Performance on the simulator improves with continuous positive airways pressure (CPAP) ${ }^{13}{ }^{15-21}$ Most of these 
studies used simple simulators with graphics which were not very realistic and the simulators have not been validated against real driving. Furthermore, subjects perform in such a way that raises questions of credibility, for example, multiple crashes and off-road events during a short run, about the relationship to real driving. ${ }^{14} 19$ This relationship is key if simulators are to be used in advising whether an individual is fit to drive. Fully immersive simulators are close to real driving but are expensive, only available in a few research centres.

The Institute for Transport Studies at the University of Leeds, UK has developed a sophisticated fully immersive driving simulator (UoLDS). This is a full size car with complex audiovisuals providing a realistic driving experience. The 'car' moves and feels real as if it is slowing down, accelerating etc. Driving simulators will never fully replicate the real driving experience, although studies have shown that there is a good correlation at the performance level. ${ }^{22}$ Driving simulators offer an alternative environment in which to study driving behaviour and hence inform road safety policy.

Alongside the full-scale simulator, a PC-based simulator (MiniSim) has been developed using the same software. The MiniSim, provides realistic graphics, incorporates steering and foot pedals and, like the UoLDS, allows continuous measurement of variables, which have been shown to relate to driver performance. $^{22} 23$

Because patients may need to perform the simulator test on more than one occasion and/or may be able to 'raise their game' if they know that their licence is at risk, it would be an advantage to assess driving performance using measures of which the patient is unaware. Therefore we have evaluated whether variables that are recorded continuously and unobtrusively on the MiniSim are associated with a one-off event that is credible as being indicative of poor driving.

\section{METHODS}

The study was conducted at St James's University Hospital, Leeds, UK. Ethical approval was obtained from the local NHS Research Ethics Committee.

\section{Patients}

Patients attending the Sleep Clinic with a confirmed diagnosis of OSAS (apnoea hypopnoea index and/or oxygen desaturation index (ODI-4\% dips in saturation) $>10 / \mathrm{h}$ ) on respiratory vari-
Table 1 Characteristics of the patient populations

\begin{tabular}{llc}
\hline Characteristics & $\begin{array}{l}\text { Exploratory study } \\
(\mathbf{n = 7 2 )}\end{array}$ & $\begin{array}{l}\text { Validation study } \\
(\mathbf{n}=\mathbf{1 3 3})\end{array}$ \\
\hline Age (years), median (IQR) & $54(46-61)$ & $52(44-60)$ \\
Men, $\mathrm{n}(\%)$ & $65(90)$ & $118(88)$ \\
$\mathrm{BMI}\left(\mathrm{kg} / \mathrm{m}^{2}\right)$, median (IOR) & $34(30-37)$ & $33(30-37)$ \\
ESS, mean (IQR) & $13(7-16)$ & $12(7-16)$ \\
AHI (events/h), median (IOR) & $32(20-54)$ & $25(13-42)$ \\
ODI (events/h), median (IOR) & $35(22-55)$ & $24(13-44)$ \\
Years since first driving licence, & $34(25-40)$ & $30(30-38)$ \\
median (IOR) & &
\end{tabular}

AHI, apnoea hypopnoea index; BMI, body mass index; ESS, Epworth Sleepiness Score; ODI, oxygen desaturation index.

able overnight sleep study (Embletta, Medcare Flaga, Reykjavik, Iceland) or overnight oximetry were approached. Recruitment was biased towards patients considered for a trial of CPAP therapy. This was to generate a patient population at risk of road traffic accident and likely to have 'events' on the simulator.

\section{Driving simulator (MiniSim)}

\section{Road layout and scenario}

A $90 \mathrm{~km}$ three-lane motorway was developed with UK standard lane markings and signage. The road is composed of eight sections (each $9 \mathrm{~km}$ in length). One section of motorway takes approximately $7 \mathrm{~min}$ to drive (at $70 \mathrm{mph}$ ) and will be referred to as one epoch. All subjects had the procedures explained and had a four-epoch practice session before commencing the test proper. A 'minor' or 'veer' event was choreographed within epoch 4 of the test run; a vehicle swerves briefly into the driver's lane, requiring an avoidance manoeuvre such as braking or swerving (or both). The vehicle is sufficiently far ahead that an alert driver should easily be able to avoid a collision. Throughout the drive, vehicles manoeuvre in and out of the subjects' lane and they react to them as they would in real life. The 'minor' event is an extension of these manoeuvres. A 'major' or 'brake' event was inserted into epoch 8 and this also signalled the end of the run. Here, a vehicle ahead brakes heavily; even with full attention some subjects might not avoid a crash.

\section{Measures and endpoints}

Task failure was defined as hitting another vehicle, veering completely out of lane (except in response to an event) or
Figure 1 Driving simulator outcomes of all patients in the exploratory phase of the study.

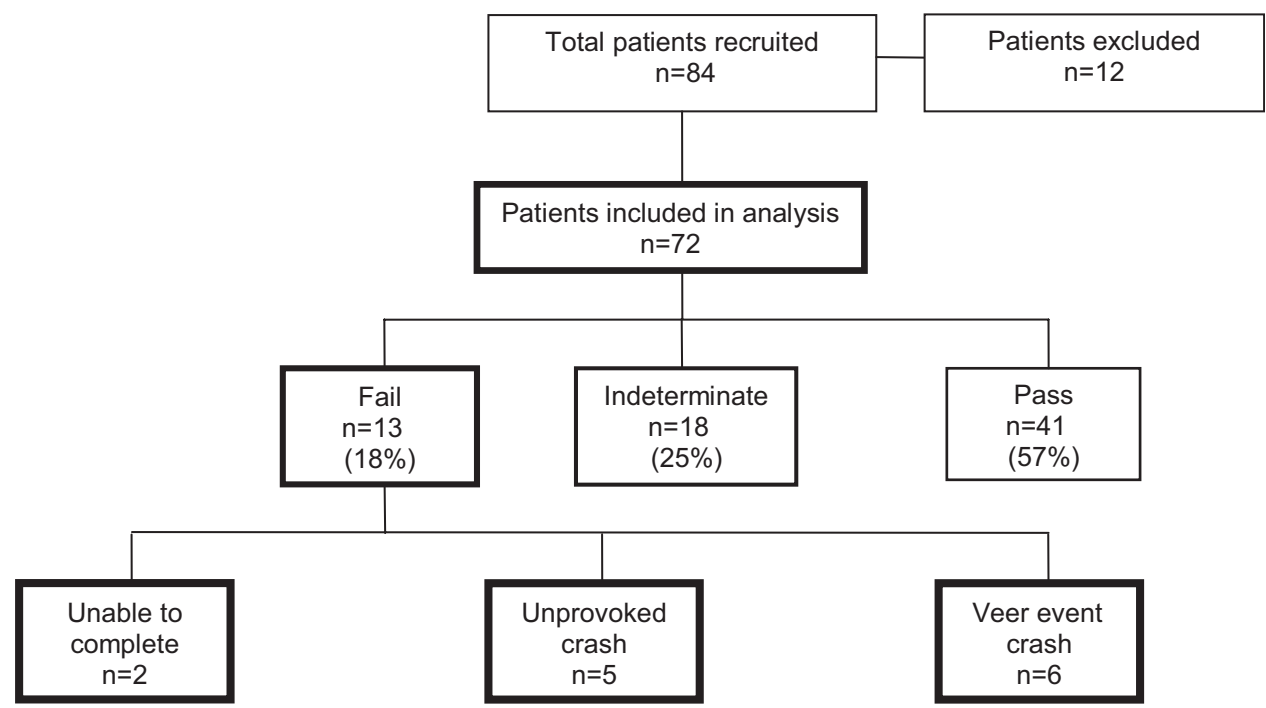


Table 2 Comparisons between the three groups in exploratory phase: fail, indeterminate and pass

\begin{tabular}{|c|c|c|c|c|c|c|c|}
\hline \multirow[b]{2}{*}{ Parameters } & \multirow{2}{*}{$\begin{array}{l}\text { Fail }(n=13) \text {, } \\
\text { mean }(S D)\end{array}$} & \multirow{2}{*}{$\begin{array}{l}\text { Indeterminate } \\
(\mathrm{n}=18) \text {, mean }(\mathrm{SD})\end{array}$} & \multirow{2}{*}{$\begin{array}{l}\text { Pass }(n=41), \\
\text { mean }(S D)\end{array}$} & \multirow{2}{*}{$\begin{array}{l}\text { One-way ANOVA } \\
\text { p Value }\end{array}$} & \multicolumn{3}{|c|}{ Bonferroni's multiple testing correction: is $p<0.05$ ? } \\
\hline & & & & & Fail vs indeterminate & Fail vs pass & Indeterminate vs pass \\
\hline SDLP 3 & $0.66(0.2)$ & $0.44(0.13)$ & $0.37(0.08)$ & $<0.001$ & Yes & Yes & No \\
\hline HFS 3 & $0.37(0.09)$ & $0.26(0.06)$ & $0.27(0.07)$ & $<0.001$ & Yes & Yes & No \\
\hline Hw1s 3 & $29.6(28.9)$ & $17.5(16.2)$ & $13.5(13)$ & 0.296 & No & No & No \\
\hline Hw 3 & $0.21(0.23)$ & $0.393(0.21)$ & $0.57(0.24)$ & $<0.001$ & No & Yes & Yes \\
\hline Speed 3 & $65(8)$ & $67(3)$ & $67(3)$ & 0.535 & No & No & No \\
\hline
\end{tabular}

BrakeRT, reaction time at the brake event; HFS 3, mean of HFS in epoch 3; Hw 3, minimum headway in epoch 3; Hw1s 3, percentage of time spent with headway under $1 \mathrm{~s}$ in epoch 3; SDLP 3 , mean SDLP in epoch 3; Speed 3, mean speed in epoch 3; TTC 3, mean of TTC in epoch 3; VeerRT, reaction time at the veer event.

spending more than $5 \%$ of the total study time $(2.5 \mathrm{~min})$ with two wheels out of the middle lane. There were four possible outcomes of the simulator runs; task failure unprovoked during the study; crashing into the vehicle in front at the 'veer' event; crashing into the vehicle in front only at the major event; no task failure at any time during the study run. Unprovoked task failure and crashes at the minor event should not happen during simulated driving and these subjects were considered to have 'failed' the test. Subjects who completed the test without meeting any of the task failure criteria defined above were deemed to have 'passed'. The subjects who only crashed at the 'brake' event were deemed to be 'indeterminate'.

The MiniSim recorded continuous measures of driving behavior, such as time it would take to collide into the lead vehicle were it to stop dead (minimum time headway, Hw), percentage time spent with minimum headway of $<1 \mathrm{~s}$ (Hw1s), minimum time to collision (TTC) to the preceding vehicle, highfrequency steering (HFS), mean speed, standard deviation of lane position (SDLP), lane changes. For the purpose of analysis we used the mean values for each parameter in epochs 3, 5, 6 and 7, which were free of events and just require steady driving at approximately $70 \mathrm{mph}$. In addition, specific measures at the programmed events were also recorded, including speed on approach to collision and reaction times (RTs).

\section{Study design and analysis}

The study was divided into two phases. In the first phase we explored whether any of the continuous (eg, SDLP, HFS, TTC, Hw1s) and event specific (eg, RT) simulator variables recorded could predict the outcomes on the MiniSim. We compared these measures of driving performance between different categories of patients using one-way analysis of variance and $t$ tests with Bonferroni's multiple testing correction. Binary logistic regression analysis was used to test the hypothesis that a 'fail' could be predicted from continuous measures of driving behaviour and thereby explore the possibility of developing a predictive model. Receiver operating characteristic (ROC) curve analyses were performed to calculate the discriminative power of the models and identify optimal cutoffs for probability score. The sensitivity, specificity and predictive powers of the models were calculated using the cutoff values. The curves generated for each model were compared using methods described by DeLong et al. ${ }^{24}$

In the second phase we validated the findings from the exploratory study in a different population. We compared ROC curves and used a two-sample z-test for comparing proportions.

Detailed methodology and definitions of simulator parameters are provided in the online supplement.

\section{RESULTS}

\section{Subject population}

Two hundred and twenty-nine patients participated in the study. Eighty-four patients were recruited for the first phase and 145 for the second. Twelve patients were excluded from each of the two phases due to inability to complete the two runs (practice and test), time constraints, simulator sickness $(n=4)$ and inability to sit continuously for $50 \mathrm{~min}$. The characteristics of the 205 patients who completed the studies are described in table 1. There were no differences between the two cohorts except that the apnoea hypopnoea index in patients in the first phase was significantly higher $(p=0.009)$.

\section{Exploratory study}

Forty-one (57\%) subjects completed the simulator runs successfully. Thirty-one (43\%) had some form of task failure (figure 1). Of the 13 subjects who failed, 9 also crashed at the brake event. Two subjects could not complete the full test run as they veered out of lane into the central reservation. One of them fell asleep.

\section{Simulator variables}

Comparisons between the three groups 'fail', 'indeterminate' and 'pass' are shown in table 2. There were significant differences in the ability to maintain lane position (figure 2), minimum time to collision with the vehicle in front (TTC), minimum time headway (Hw), HFS (figure 3 ) and RT at the veer and brake events (figure 4). These significant differences were

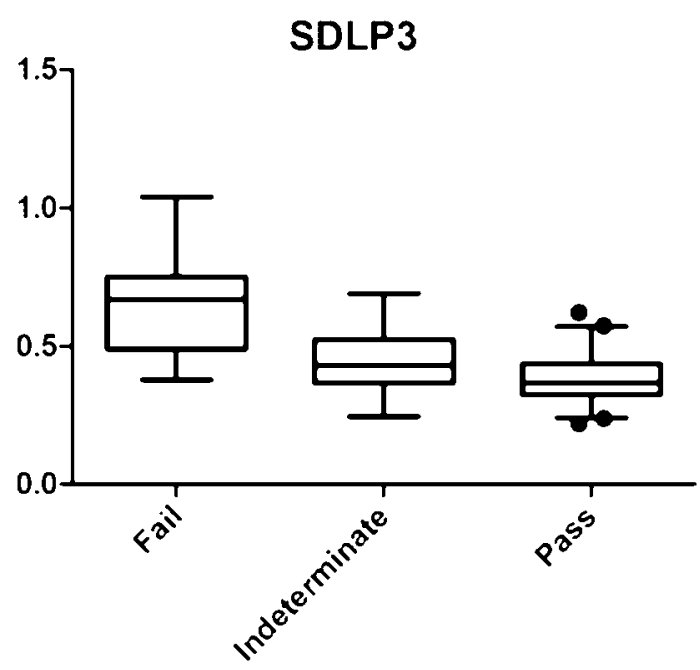

Figure 2 Distribution of mean of SD of lane position (SDLP) in epoch 3 between the three groups. 


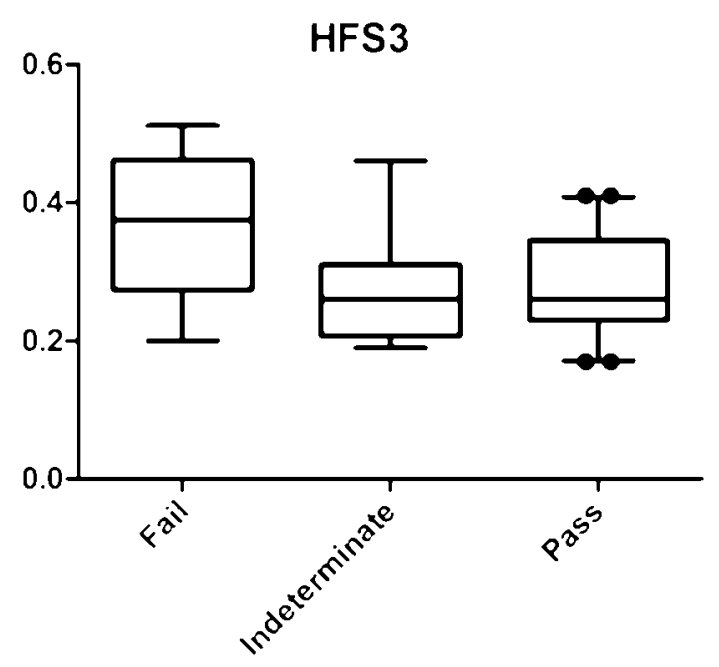

Figure 3 Distribution of mean of proportion of high-frequency steering activity (HFS) in epoch 3 between the three groups.

maintained irrespective of the epochs (3, 5, 6 or 7$)$ used and therefore, for simplicity, ease of presentation and because this may allow the test to be shortened in future, only data from epoch 3 are presented and used in the regression analyses. There was a clear distinction between 'fails' and the rest, but only the VeerRT and Hw 3 were significantly different between the 'pass' and 'indeterminate' groups.

\section{Regression analysis and ROC curves}

Two predictive models emerged in differentiating 'fails' from the others. Model 1-including only the SDLP 3, and Model 2 -includes a combination of SDLP 3 and VeerRT.

Model 2 had higher predictive power. Figure 5 compares the two models using ROC curve analysis and reinforces the finding from the regression analysis. The first model has an area under the curve $(\mathrm{AuC})$ of 0.89 and the second 0.93 . The difference between the two ROC curves was not significant $(p=0.132)$. Table 3 compares the sensitivities and specificities of the two models at the chosen cutoffs. The chosen cutoffs $(0.15$ for model 1 and 0.3 for model 2) quoted here are a compromise between extremes of high sensitivity and high specificity, giving equal weight to both.

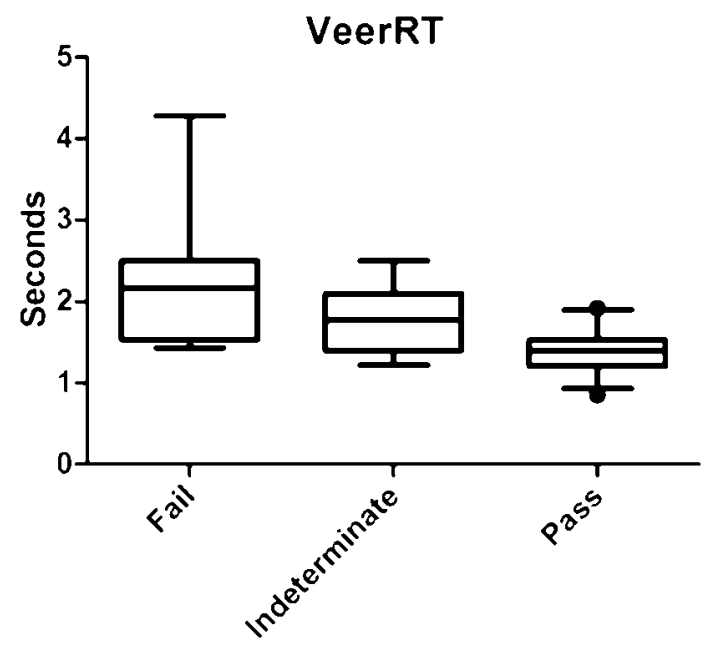

Figure 4 Distribution of mean veer reaction time (VeerRT) between the three groups.

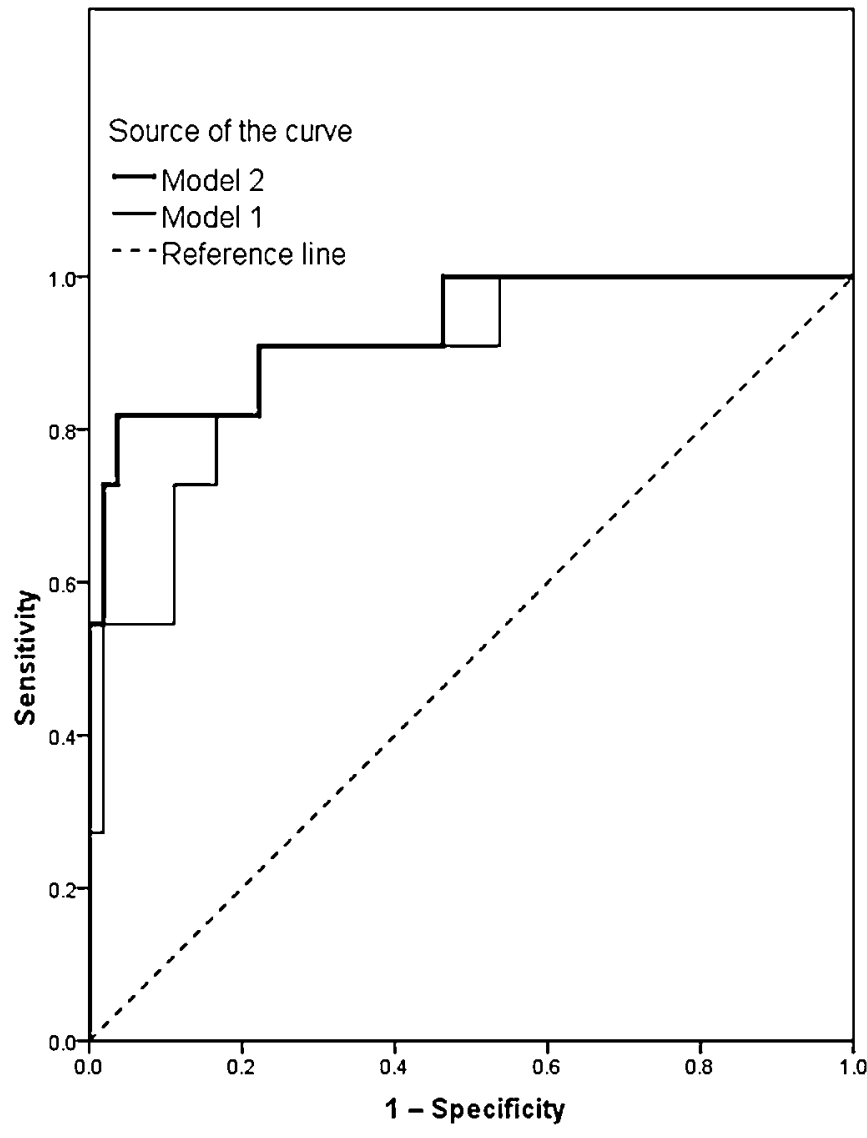

Figure 5 Receiver operating characteristic curves for the two models.

The details of the extremes of cutoffs, regression coefficients and equations are provided in the online supplement.

A similar regression analysis was performed excluding those who had 'failed' to distinguish between 'indeterminates' and 'passes'. AuC in the ROC curve analysis was 0.84 . The sensitivity of that model to identify 'pass' was 97\% (95\% CI 86\% to 99\%) with 53\% (95\% CI 26\% to 79\%) specificity; VeerRT and Hw were the parameters in the equation. The positive predictive value was $84 \%$ (95\% CI $70 \%$ to $93 \%$ ) and the negative predictive value only $88 \%$ (95\% CI $51 \%$ to $99 \%$ ) using a cutoff of 0.5 . Hence using simulator data the 'failed' group could be predicted with much greater certainty than the 'indeterminate' group.

\section{Validation study}

One hundred and thirty-three patients were analysed for the validation study and the outcomes are described in figure 6. Most subjects who failed fulfilled more than one task failure criterion. Of the 13 subjects who could not complete the full run, 4 also had an unprovoked crash and 4 crashed at the veer event. Nine of

Table 3 Comparing optimum sensitivities and specificities of the two models

\begin{tabular}{lllll}
\hline Models & Sensitivity (\%) & Specificity (\%) & $\begin{array}{l}\text { Positive } \\
\text { predictive } \\
\text { value (\%) }\end{array}$ & $\begin{array}{l}\text { Negative } \\
\text { predictive } \\
\text { value (\%) }\end{array}$ \\
\hline $\begin{array}{l}\text { Model } 1 \\
\text { (cutoff } 0.15)\end{array}$ & 76 (46 to 95) & $84(72$ to 92) & $53(25$ to 75$)$ & 94 (84 to 99) \\
$\begin{array}{l}\text { Model } 2 \\
\text { (cutoff } 0.3)\end{array}$ & 82 (48 to 98) & 96 (87 to 99) & 82 (48 to 98) & 96 (87 to 99) \\
\hline
\end{tabular}

Note: $95 \% \mathrm{Cl}$ 
Figure 6 Simulator outcomes of the validation phase of the study.

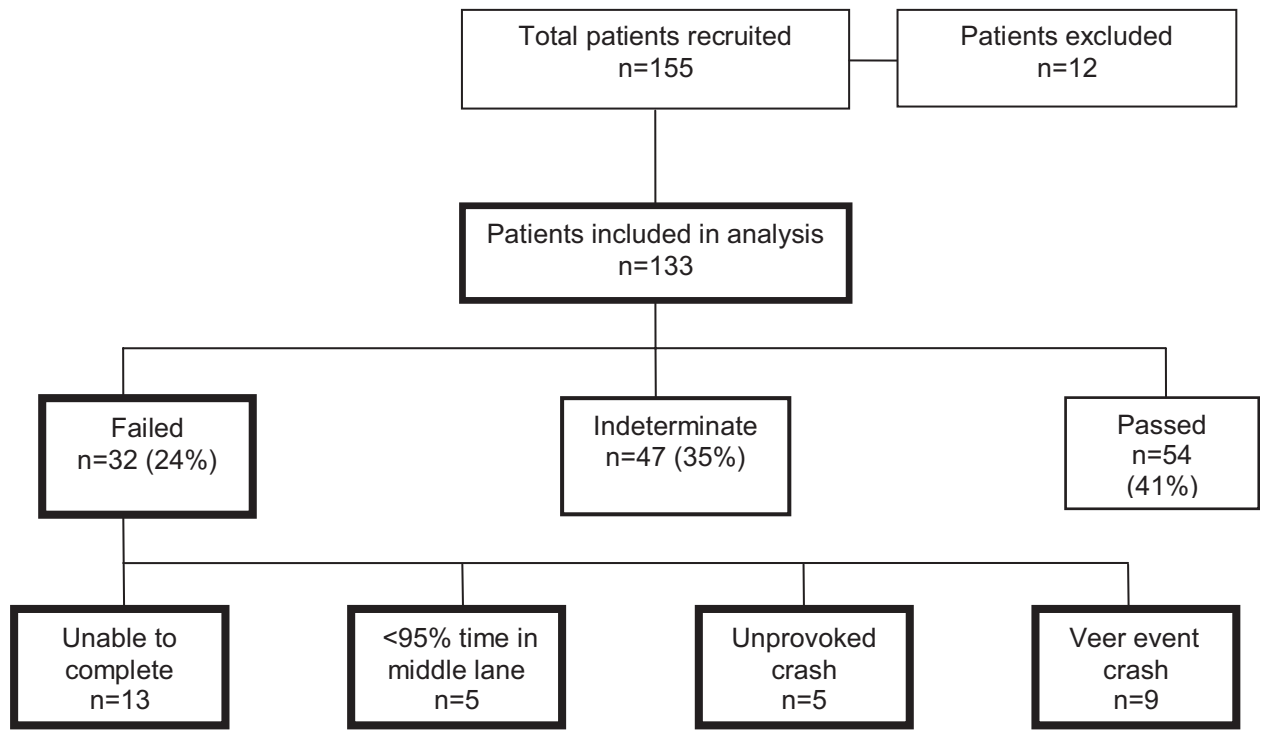

them also failed on lane criteria. Of the 19 failed subjects who completed the full run, 12 also crashed at the brake event.

Table 4 compares the three categories. The results are similar to the first phase of the study, broadly showing the same relationship between the simulator parameters.

We could apply the equation from model 1 to 133 subjects and model 2 to 113 subjects due to unavailability of either of the two parameters; some subjects did not brake at the veer event and avoided a crash just by swerving; others crashed before epoch 3 and hence SDLP 3 was not available. A ROC curve was constructed with the predicted probability score for model 2 generated by the equation derived in the exploratory study for each subject. The AuC was 0.9 compared with 0.93 in the exploratory study. The difference between the two curves was not significant $(p=0.570)$. Furthermore the calculated sensitivities and specificities at the chosen cutoffs were not significantly different between the two cohorts when compared using z-tests, confirming that the findings of the exploratory study are valid in a different population (table 5).

\section{DISCUSSION}

We have shown in two different cohorts that with the MiniSim it is possible to distinguish, with a high degree of confidence, patients with OSAS who unequivocally crash during simulated driving from those who are able to complete a $50 \mathrm{~min}$ drive without incident. We can also identify a group, but with less confidence, whose performance is intermediate. Not only was their response at the veer event different from the others but there was a clear hierarchical pattern for other parameters
(SDLP, HFS, Hw and BrakeRT); subjects who 'failed' were worst, those who 'passed' the best, with the 'indeterminates' in the middle.

The sensitivities, specificities and the predictive values can be calculated for different cutoffs; the one chosen will depend upon the attitude to risk. At one extreme, all accidents should be prevented and anybody with the slightest possibility of having an accident should be identified. At the other extreme, the emphasis will be on being as sure as possible that an individual is an unsafe driver. We have quoted compromise values for cutoffs, giving equal weight to sensitivity and specificity; others might choose a different value. Examples are given in the online supplement.

We deliberately tried to recruit patients at risk of having problems driving; many completed a 50 min run on a realistic motorway without incident. This can potentially overestimate the sensitivities and specificities compared with a general OSAS cohort. However, in real life this test would probably be used on patients with OSAS who are considered to be high risk. One problem with previous simulator studies was the number of crashes or events during the study. In a study by Juniper et al ${ }^{19}$ patients with OSAS had a median of 5.2 off-road events per hour. Similarly Turkington et $a l^{14}$ reported an average of 24 offroad events per hour in their study using a divided attention driving simulator. Even patients with severe OSAS do not have multiple events during 20 min of on-road driving. The criteria that we used for 'fail' are realistic and understandable to patients. This is very important if the test is to have credibility; an individual who fails on the simulator because they go off road

Table 4 Comparisons between the three groups in validation study: fail, indeterminate and pass

\begin{tabular}{|c|c|c|c|c|c|c|c|}
\hline \multirow[b]{2}{*}{ Parameters } & \multirow{2}{*}{$\begin{array}{l}\text { Fail }(n=32) \text {, } \\
\text { mean (SD) }\end{array}$} & \multirow{2}{*}{$\begin{array}{l}\text { Indeterminate } \\
(\mathrm{n}=47) \text {, mean }(\mathrm{SD})\end{array}$} & \multirow{2}{*}{$\begin{array}{l}\text { Pass }(n=54) \text {, } \\
\text { mean (SD) }\end{array}$} & \multirow{2}{*}{$\begin{array}{l}\text { One-way ANOVA } \\
\text { p Value }\end{array}$} & \multicolumn{3}{|c|}{ Bonferroni's multiple testing correction: is $p<0.05$ ? } \\
\hline & & & & & Fail vs indeterminate & Fail vs pass & Indeterminate vs pass \\
\hline SDLP 3 & $0.55(0.2)$ & $0.42(0.1)$ & $0.38(0.09)$ & $<0.001$ & Yes & Yes & No \\
\hline HFS 3 & $0.35(0.09)$ & $0.3(0.08)$ & $0.26(0.08)$ & 0.001 & Yes & Yes & No \\
\hline Hw 3 & $0.30(0.22)$ & $0.48(0.27)$ & $0.59(0.19)$ & $<0.001$ & No & Yes & Yes \\
\hline TाС 3 & $3.19(3.2)$ & $8(18)$ & $7.1(7.2)$ & 0.195 & No & No & No \\
\hline VeerRT (s) & $2.2(0.55)$ & $1.57(0.45)$ & $1.44(0.34)$ & $<0.001$ & Yes & Yes & No \\
\hline BrakeRT (s) & $3.27(1)$ & $3.5(0.8)$ & $2.29(0.9)$ & $<0.001$ & No & Yes & Yes \\
\hline
\end{tabular}

BrakeRT, reaction time at the brake event; HFS 3, mean of HFS in epoch 3; Hw 3, minimum headway in epoch 3; SDLP 3, mean SDLP in epoch 3; TTC 3, mean of TTC in epoch 3; VeerRT, reaction time at the veer event. 
Table 5 Sensitivities, specificities and predictive values of the two models at chosen cutoffs applied to the exploratory and validation cohorts and $p$ values for a two-sample z-test comparing the sensitivities and specificities of the chosen cutoffs in the two populations

\begin{tabular}{|c|c|c|c|c|}
\hline Models & Sensitivity & Specificity & $\begin{array}{l}\text { Positive } \\
\text { predictive } \\
\text { value }\end{array}$ & $\begin{array}{l}\text { Negative } \\
\text { predictive } \\
\text { value }\end{array}$ \\
\hline \multicolumn{5}{|l|}{ Model 1 (cutoff 0.15) } \\
\hline Exploratory cohort & 76 (46 to 95 ) & 84 (72 to 92 ) & 53 (25 to 75$)$ & 94 (84 to 99 ) \\
\hline Validation cohort & 60 (41 to 77 ) & 85 (77 to 91 ) & 55 (36 to 72 ) & 88 (80 to 93 ) \\
\hline$p$ Values for z-test & 0.507 & 0.866 & & \\
\hline \multicolumn{5}{|l|}{ Model 2 (cutoff 0.3 ) } \\
\hline Exploratory cohort & 82 (48 to 98 ) & 96 (87 to 99 ) & 82 (48 to 98 ) & 96 (87 to 99 ) \\
\hline Validation cohort & 60 (36 to 81 ) & 93 (85 to 97 ) & 63 (38 to 84 ) & 92 (84 to 96$)$ \\
\hline$p$ Values for z-test & 0.384 & 0.342 & & \\
\hline
\end{tabular}

Note: $95 \% \mathrm{Cl}$.

multiple times might argue, quite reasonably, that this is not what happens when they drive a real car and therefore that the simulation is not valid.

While it might be reasonable to include an event such as our final 'brake' event, as failure to avoid this is realistic evidence of sub-optimal performance, it has the disadvantage that it may limit the usefulness of the test for repeated use. Longitudinal studies have found that behaviour adapts and changes over time, in driving simulator experiments. ${ }^{25}$ Likewise, a patient expecting something may perform differently on subsequent occasions. ${ }^{26}$ Furthermore, a patient may drive poorly at other times during the test but perform adequately at the event. Variables that are recorded continuously throughout, and of which the patient is unaware, are preferable. In common with previous studies we found that poor lane control (SDLP) was predictive of a crash. ${ }^{18} 19$ Predictive power was increased by the inclusion of reaction time at the veer event. Again previous studies have shown that patients with OSAS who are untreated have worse reaction times than controls and patients with OSAS after CPAP therapy. ${ }^{18} 19$ This is likely to be an underestimate as we had to exclude some patients from the analysis; some subjects $(n=5)$ did not brake at all at the veer event and avoided a crash by veering out of lane, a legitimate manoeuvre; others $(n=4)$ did not brake at all and crashed. Although this assessment requires an 'event' it was a subtle extension of routine driving behaviour and is unlikely to be memorable.

Though we have explored hard endpoints there must be scope for the clinician to make decisions on an individual basis. Subjects who visibly struggle to stay awake, utilise various coping strategies to stay awake (eg, one subject sang and thumped the desk throughout the test), should not necessarily be deemed to have passed. Alternatively, subjects who show good lane control but happen to crash due to a momentary lapse might still be considered to have passed. This second group are the subjects who could not be identified correctly by the regression analysis. The 'indeterminate' group warrant further study; while it can be argued that failure at the brake event alone does not necessarily indicate unsafe driving, their performance across a wide range of measures was clearly worse than those who passed.

Many questions still need to be answered before the MiniSim, or similar advanced simulators, can be used to help advise patients with OSAS about driving, but it does hold promise. It has significant advantages over previously described simulators in terms of realism and results that are credible in terms of their relationship to normal driving. That events can be reliably predicted from parameters of which the patient is unaware is an advantage. An objective test is an advance over the current situation in which inconsistent advice is given, based upon unreliable data, coloured by the clinician's individual stance on driving and accident risk. The work described here is the first step towards the development of an objective test that could have a major impact on the reliability of the advice provided.

Acknowledgements We are grateful to Michael Daly, Tony Horrobin and Hamish Jamson from the Institute for Transport Studies for their contribution to the development and running of the simulator. We are thankful to the contributions of research nurse Craig Armstrong, sleep physiologist Sue Watts, specialist nurses Martin Latham, Jampa Choedon, Mitchell Nix, Lisa Emmett, healthcare assistant Anne Kellett and Audrey Rowe and Susan Leigh from the Sleep Services administrative team.

Contributors DG: design of study, data collection, analysis, writing the paper. SLJ: design of study, analysis, writing the paper. PDB: design, statistical analysis, writing the paper. MWE: original concept, design of study, writing the paper.

Funding The study was partly supported by an unrestricted grant from ResMed for healthcare assistant time to supervise simulator runs.

Competing interests None

Patients consent Obtained

Ethics approval NHS Research Ethics Committee, York.

Provenance and peer review Not commissioned; externally peer reviewed.

\section{REFERENCES}

1. Arand D, Bonnet M, Hurwitz T, et al. The clinical use of the MSLT and MWT. Sleep 2005;28:123-44

2. Carskadon MA, Dement WC. Multiple sleep latency tests during the constant routine. Sleep 1992;15:396-9.

3. Engleman HM, Hirst WS, Douglas NJ. Under reporting of sleepiness and driving impairment in patients with sleep apnoea/hypopnoea syndrome. J Sleep Res 1997;6:272-5.

4. Reimer B, D'Ambrosio LA, Coughlin JE, et al. Using self-reported data to assess the validity of driving simulation data. Behav Res Methods 2006;38:314-24.

5. Ingre M, Akerstedt $T$, Peters $B$, et al. Subjective sleepiness and accident risk avoiding the ecological fallacy. J Sleep Res 2006;15:142-8.

6. Liu GF, Han S, Liang DH, et al. Driver sleepiness and risk of car crashes in Shenyang, a Chinese northeastern city: population-based case-control study. Biomed Environ Sci 2003:16:219-26.

7. Teran-Santos J, Jimenez-Gomez A, Cordero-Guevara J. The association between sleep apnea and the risk of traffic accidents. Cooperative Group Burgos-Santander. N Engl J Med 1999;340:847-51

8. Bennett LS, Stradling JR, Davies RJ. A behavioural test to assess daytime sleepiness in obstructive sleep apnoea. J Sleep Res 1997;6:142-5.

9. Punjabi NM, Bandeen-Roche K, Young T. Predictors of objective sleep tendency in the general population. Sleep 2003;26:678-83.

10. Sangal RB, Sangal JM, Belisle C. Subjective and objective indices of sleepiness (ESS and MWT) are not equally useful in patients with sleep apnea. Clin Electroencephalogr 1999;30:73-5.

11. Morrell MJ, Jackson ML, Twigg GL, et al. Changes in brain morphology in patients with obstructive sleep apnoea. Thorax 2010;65:908-14.

12. Findley LJ, Suratt PM, Dinges DF. Time-on-task decrements in 'steer clear' performance of patients with sleep apnea and narcolepsy. Sleep 1999;22 $804-9$.

13. George CF, Boudreau AC, Smiley A. Simulated driving performance in patients with obstructive sleep apnea. Am J Respir Crit Care Med 1996;154:175-81.

14. Turkington PM, Sircar M, Allgar V, et al. Relationship between obstructive sleep apnoea, driving simulator performance, and risk of road traffic accidents. Thorax 2001;56:800-5.

15. Findley LJ, Fabrizio MJ, Knight $\mathrm{H}$, et al. Driving simulator performance in patients with sleep apnea. Am Rev Respir Dis 1989;140:529-30.

16. George CF. Reduction in motor vehicle collisions following treatment of sleep apnoea with nasal CPAP. Thorax 2001:56:508-12.

17. Hack M, Davies RJ, Mullins R, et al. Randomised prospective parallel trial of therapeutic versus subtherapeutic nasal continuous positive airway pressure on simulated steering performance in patients with obstructive sleep apnoea. Thorax 2000:55:224-31.

18. Hack MA, Choi SJ, Vijayapalan P, et al. Comparison of the effects of sleep deprivation, alcohol and obstructive sleep apnoea (OSA) on simulated steering performance. Respir Med 2001;95:594-601

19. Juniper M, Hack MA, George CF, et al. Steering simulation performance in patients with obstructive sleep apnoea and matched control subjects. Eur Respir J 2000;15:590-5. 
20. Orth M, Leidag M, Kotterba S, et al. [Estimation of accident risk in obstructive sleep apnea syndrome (OSAS) by driving simulation] (In German). Pneumologie 2002; 56:13-18.

21. Turkington PM, Sircar M, Saralaya D, et al. Time course of changes in driving simulator performance with and without treatment in patients with sleep apnoea hypopnoea syndrome. Thorax 2004:59:56-9.

22. Carsten OMJ, Groeger JA, Blana E, et al. Driver performance in the engineering and physical sciences research council driving simulator: a validation study. Driver Performance in the Engineering and Physical Sciences Research Council Driving Simulator. Engineering and Physical Sciences Research Council, UK, 1997. Report No. GR/K56162.
23. Blana $\mathbf{E}$, Golias J. Differences between vehicle lateral displacement on the road and in a fixed-base simulator. Hum Factors 2002;44:303-13.

24. DeLong ER, DeLong DM, Clarke-Pearson DL. Comparing the areas under two or more correlated receiver operating characteristic curves: a nonparametric approach Biometrics 1988;44:837-45.

25. Jamson S, Lai $F$, Jamson H. Driving simulators for robust comparisons: a case study evaluating road safety engineering treatments. Accid Anal Prev 2010;42:961-71.

26. Engstrom J, Aust ML, Vistrom M. Effects of working memory load and repeated scenario exposure on emergency braking performance. Hum Factors 2010;52:551-9.

\section{Journal club}

\section{Chronic disease management for tobacco dependence}

This randomised control trial compared the efficacy of utilising chronic disease management principles for tobacco dependence using a tailored intervention with standard care. As tobacco dependence is a chronic relapsing condition, the tailored intervention was chosen to account for possible interim setbacks.

Four hundred and forty-three eligible participants received five telephone-counselling calls and 4 weeks of nicotine replacement therapy. They were randomised to receive continuing counselling and nicotine replacement therapy for 1 year (longitudinal care, LC) or to receive one additional call at 8 weeks (evidence-based usual care, UC). The primary outcome was 6 months of prolonged abstinence, measured at 18 months following initial quit date. Secondary outcomes included abstinence rates before 6 months and smoking reduction.

At 18 months, $30.2 \%$ of LC participants reported 6 months of abstinence from smoking, compared with $23.5 \%$ in UC. Prior to 6 months, abstinence rates were slightly higher with UC than LC. At all time points, those who did not quit had greater smoking reduction with LC than UC (statistically significant only at 12 months). With LC, quit rates rose throughout the year without reaching a plateau, suggesting that extending treatment further may be beneficial.

One limitation of this study was the difficulty in differentiating between the effects of behavioural and medical treatment. Results were not biochemically confirmed, but the study population was believed to be low-risk for incorrectly reporting smoking status. The LC model allowed counsellors to adjust treatment in response to smokers' experiences of quitting and to positively reinforce the option of interim smoking reduction. Chronic management appears to be a feasible approach to increase long-term abstinence.

- Joseph AM, Fu SS, Lindgren B, et al. Chronic disease management for tobacco dependence. Arch Intern Med 2011;171:1894-900.

\section{Harshani M Chandrasekera}

Correspondence to Dr Harshani M Chandrasekera, FY2, Department of Thoracic Medicine, The Royal Free Hospital, Pond Street, London NW3 20G, UK; harshani.chandrasekera@nhs.net

Published Online First 27 January 2012

Thorax 2012;67:821. doi:10.1136/thoraxjnl-2012-201586 